\title{
Step Bunching with Alternation of Structural Parameters
}

\author{
Masahide Sato* Makio Uwaha ${ }^{a}$, Tomonori Mori, and Yukio Hirose \\ Department of Computational Science, Kanazawa University, \\ Kakuma-machi, Kanazawa 920-1192, Japan \\ ${ }^{a}$ Department of Physics, Nagoya University, \\ Furo-cho, Chikusa-ku, Nagoya 464-8602, Japan
}

\begin{abstract}
By taking account of the alternation of structural parameters, we study bunching of impermeable steps induced by drift of adatoms on a vicinal face of $\mathrm{Si}(001)$. With the alternation of diffusion coefficient, the step bunching occurs irrespective of the direction of the drift if the step distance is large. Like the bunching of permeable steps, the type of large terraces is determined by the drift direction. With step-down drift, step bunches grows faster than those with step-up drift. The ratio of the growth rates is larger than the ratio of the diffusion coefficients. Evaporation of adatoms, which does not cause the step bunching, decreases the difference. If only the alternation of kinetic coefficient is taken into account, the step bunching occurs with step-down drift. In an early stage, the initial fluctuation of the step distance determines the type of large terraces, but in a late stage, the type of large terraces is opposite to the case of alternating diffusion coefficient.

PACS numbers: $81.10 . \mathrm{Aj}, 05.70 . \mathrm{Ln}, 47.20 . \mathrm{Hw}, 68.35 . \mathrm{Fx}$
\end{abstract}

\section{INTRODUCTION}

When a $\mathrm{Si}(001)$ vicinal face is tilted in the $\langle 110\rangle$ direction, two types of terraces, $1 \times 2$ and $2 \times 1$ terraces, appear alternately. In the $1 \times 2$ terrace, which we call $\mathrm{T}_{\mathrm{A}}$, the surface diffusion perpendicular to the steps is faster than that parallel to the steps. In the $2 \times 1$ terrace, which we call $\mathrm{T}_{\mathrm{B}}$, the relation is opposite.

These terraces are separated by single steps. The type of the steps also changes alternately: the step at the upper side edge of $\mathrm{T}_{\mathrm{B}}$, which we call $\mathrm{S}_{\mathrm{B}}$, is rougher than that of $\mathrm{T}_{\mathrm{A}}$, which we call $\mathrm{S}_{\mathrm{A}}$. Then, parameters like kinetic coefficient and the step stiffness may change for the two kinds of steps.

When a $\mathrm{Si}(001)$ vicinal face is heated by direct electric current, the vicinal face is unstable and step bunching occurs irrespective of the current direction [1, 2]. The type of large terraces between bunches is $\mathrm{T}_{\mathrm{A}}$ with stepdown current and $\mathrm{T}_{\mathrm{B}}$ with step-up current. Cause of the step bunching is considered to be the drift of adatoms [3] induced by the current. By using a one-dimensional step model, where the alternation of diffusion coefficient and that of kinetic coefficient are taken into account, Stoyanov [4] theoretically studied the stability of a vicinal face for pairing of steps. With large kinetic coefficients, the step pairing occurs irrespective of the direction of the drift, and the type of large terraces between step pairs is determined by the current direction. To study behaviors of step pairs, Natori and co-workers carried out numerical simulation of a similar one-dimensional step model [5, 6]. With step-down drift, the step bunching occurs via coalescence of step pairs, but the step bunching does not

\footnotetext{
* Present address: Imformation Media Center of Kanazawa Univer-
} sity.; Electronic address: sato@cs.s.kanazawa-u.ac.jp occur with step-up drift, which disagrees with the experiments [1, 2].

Recently, we carried out Monte Carlo simulation [7], where the alternation of anisotropic surface diffusion was taken into account. In contrast to the previous studies [5, 6], the step bunching occurs irrespective of the direction of the drift as in the experiment 1, 2]. In our model [7], the steps are perfectly permeable, and the alternation of kinetic coefficient and the evaporation of adatoms are neglected. On the other hand, the steps are impermeable and both the alternation of kinetic coefficient and the evaporation are taken into account in the previous models [5, 6]. Since there are many differences between the models, it is not clear what is the most important factor to cause the different results.

In this paper, we use a one-dimensional model of impermeable steps to study the drift-induced step bunching. In Sec. II we introduce the model. In Sec. III we analyze the model with alternating diffusion coefficient and perform a numerical simulation. We compare the results with our previous study [7]. In Sec. IV] we study the model with the alternating kinetic coefficient. The effect of evaporation, which is neglected in Ref. 7, is also studied in Secs. III and IV In Sec. V we summarize the results and give a brief discussion.

\section{MODEL}

We use a one-dimensional step flow model [4, 5, 6]. The $y$-coordinate is taken in the step-down direction. When the drift of adatoms is parallel to the $y$-axis, the diffusion equation of adatom density $c(y, t)$ is given by

$$
\frac{\partial c}{\partial t}=D_{m} \frac{\partial^{2} c}{\partial y^{2}}-\frac{D_{m} F}{k_{\mathrm{B}} T} \frac{\partial c}{\partial y}-\frac{1}{\tau} c
$$


where $D_{m}$ is the diffusion coefficient in the $m$ th terrace, $F$ the force to cause the drift and $\tau$ the lifetime of adatoms for evaporation.

Boundary conditions at the $m$ th step are given by $[4,8]$

$$
\begin{aligned}
K_{m}\left(\left.c\right|_{+}-c_{m}\right)= & D_{m}\left(\left.\frac{\partial c}{\partial y}\right|_{+}-\left.\frac{F}{k_{\mathrm{B}} T} c\right|_{+}\right) \\
& -P_{m}\left(\left.c\right|_{+}-\left.c\right|_{-}\right), \\
K_{m}\left(\left.c\right|_{-}-c_{m}\right)= & -D_{m-1}\left(\left.\frac{\partial c}{\partial y}\right|_{-}-\left.\frac{F}{k_{\mathrm{B}} T} c\right|_{-}\right) \\
& -P_{m}\left(\left.c\right|_{-}-\left.c\right|_{+}\right),
\end{aligned}
$$

where $c_{m}$ is the equilibrium adatom density, $K_{m}$ kinetic coefficient of the $m$ th step and $+(-)$ indicates the lower (upper) side of the step. $\mathrm{T}_{\mathrm{A}}$ and $\mathrm{T}_{\mathrm{B}}$ appear alternately, and we assume steps with odd numbers are $S_{\mathrm{B}}$ steps and those with even numbers are $S_{\mathrm{A}}$ steps (Figure 11). The diffusion coefficients and the kinetic coefficients are given by $\left(D_{2 n}, K_{2 n}\right)=\left(D_{\mathrm{A}}, K_{\mathrm{A}}\right)$ and $\left(D_{2 n-1}, K_{2 n-1}\right)=\left(D_{\mathrm{B}}, K_{\mathrm{B}}\right)$. Bearing a $\mathrm{Si}(001)$ vicinal face in mind, we set $D_{A}>D_{B}$ and $K_{B}>K_{A}$. The second terms in the right hand side of eqs. (2) and (3) represent the adatom current through the step without solidification. With $P_{m} \rightarrow \infty$, the difference between $\left.c\right|_{+}$and $\left.c\right|_{-}$vanishes and the step is called perfectly permeable. With $P_{m}=0$, the step is called impermeable and the surface diffusion field is separated by the step. Hereafter we deal with the impermeable steps.

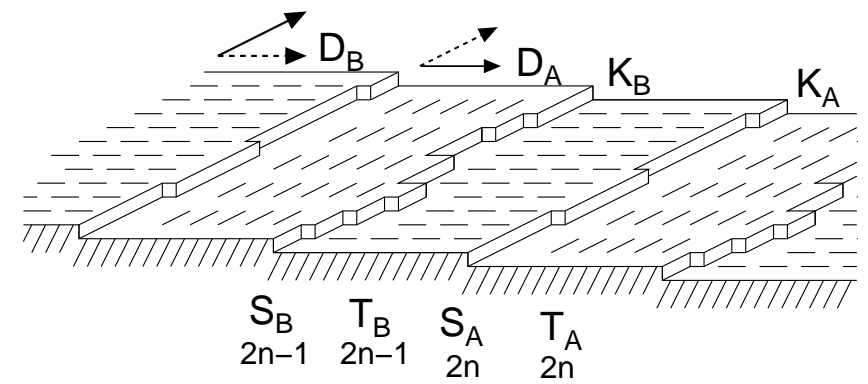

FIG. 1: A restructed $\mathrm{Si}(001)$ vicinal face. Short lines represent dimers.

When the neighboring steps interact with the potential $\zeta_{m}$, the equilibrium adatom density at the $m$ th step $c_{m}$ is given by [9, 10]

$$
\begin{aligned}
c_{m}= & c_{\mathrm{eq}}^{0}+\frac{\Omega c_{\mathrm{eq}}^{0}}{k_{\mathrm{B}} T} \frac{\partial \zeta_{m}}{\partial y_{m}} \\
& =c_{\mathrm{eq}}^{0}\left[1-\tilde{A}_{\tilde{\nu}}\left(\frac{1}{l_{m-1}^{\tilde{\nu}}}-\frac{1}{l_{m}^{\tilde{\nu}}}\right)\right]
\end{aligned}
$$

where $c_{\mathrm{eq}}^{0}$ is the equilibrium adatom density of an isolated step, $\Omega$ the atomic area, $y_{m}$ the position of the $m$ th step and $l_{m}=\left(y_{m+1}-y_{m}\right)$ is the width of the $m$ th terrace. If the step interaction potential $\zeta_{m}$ is given by $\zeta_{m}=$
$-A\left(\ln l_{m-1}+\ln l_{m}\right)$ as in a $\operatorname{Si}(001)$ vicinal face [1], the exponent is $\tilde{\nu}=1$ and $\tilde{A}_{\tilde{\nu}}=\Omega A / k_{\mathrm{B}} T$.

By solving eq. (11) with the boundary conditions, eqs. (2) and (3), in a quasi-static approximation $(\partial c / \partial t=$ $0)$, we determine the adatom density. The velocity of the $m$ th step is given by

$$
\begin{aligned}
V_{m}= & K_{m}\left(\left.c\right|_{+}-c_{m}\right)+K_{m}\left(\left.c\right|_{-}-c_{m}\right) \\
= & \Omega\left(\left.D_{m} \frac{\partial c}{\partial y}\right|_{+}-\left.\frac{D_{m} F}{k_{\mathrm{B}} T} c\right|_{+}\right) \\
& -\Omega\left(\left.D_{m-1} \frac{\partial c}{\partial y}\right|_{-}-\left.\frac{D_{m-1} F}{k_{\mathrm{B}} T} c\right|_{-}\right) .
\end{aligned}
$$

In the following, to see how the step bunching changes with the alternation of parameters, we separately study the effect of diffusion coefficients and that with kinetic coefficients.

\section{STEP BUNCHING WITH ALTERNATION OF THE DIFFUSION COEFFICIENT}

We use the model of impermeable steps to study the step bunching with alternation of diffusion coefficient. We compare results wtih the previous study [7] to see the effect of step permeability. For simplicity, the alternation of kinetic coefficient is neglected.

\section{A. Step bunching induced by the drift in a conserved system}

We first study the step bunching in a conserved system. Without the evaporation, the step velocity is given by

$$
\begin{aligned}
V_{m}= & \frac{\Omega K D_{m-1} f\left(c_{m-1} e^{f l_{m-1}}-c_{m}\right)}{\left(e^{f l_{m-1}}-1\right) K+\left(e^{f l_{m-1}}+1\right) D_{m-1} f} \\
& -\frac{\Omega K D_{m} f\left(c_{m} e^{f l_{m}}-c_{m+1}\right)}{\left(e^{f l_{m}}-1\right) K+\left(e^{f l_{m}}+1\right) D_{m} f}
\end{aligned}
$$

where $f=F / k_{\mathrm{B}} T$.

In a vicinal face with the step distance $l$, the step velocities are given by

$$
\begin{aligned}
V_{2 n} & =-V_{2 n-1} \\
& =\frac{\Omega K^{2} f c_{\mathrm{eq}}^{0}\left(e^{f l}-1\right)^{2}\left(D_{\mathrm{B}}-D_{\mathrm{A}}\right)}{v_{A} v_{B}},
\end{aligned}
$$

where $v_{A}$ and $v_{B}$ are

$$
\begin{aligned}
& v_{A}=\left(e^{f l}-1\right) K+\left(e^{f l}+1\right) D_{\mathrm{A}} f, \\
& v_{B}=\left(e^{f l}-1\right) K+\left(e^{f l}+1\right) D_{\mathrm{B}} f .
\end{aligned}
$$

Since the diffusion coefficient $D_{\mathrm{A}}$ is larger than $D_{\mathrm{B}}, S_{\mathrm{A}}$ steps advance and $S_{\mathrm{B}}$ steps recede with step-down drift $(f>0)$. With step-up drift $(f<0)$, the direction of the step motion is reversed. 
In the initial stage of the instability, formation of large $\mathrm{T}_{\mathrm{A}}\left(\mathrm{T}_{\mathrm{B}}\right)$ terrace with step-down (step-up) drift is expected due to the step pairing. To study the motion of pairs of steps, we carry out numerical integration of eq. (6). In addition to the step distance $l$, there are two characteristic lengths in the vicinal face. One is $f^{-1} \sim k_{\mathrm{B}} T / e E[12$, which is a characteristic length determined by the drift and usually much larger than the step distance. The other is $D_{\mathrm{A}} / K$, which is characteristic length determined by step kinetics. When the step distance $l$ is much smaller than $D_{\mathrm{A}} / K$, the step kinetics is more important than the surface diffusion. We assume that $f^{-1}$ is larger than $D_{\mathrm{A}} / K$ and $l$, and study the time evolution in two cases; $D_{\mathrm{A}} / K<l$ and $l<D_{\mathrm{A}} / K$.

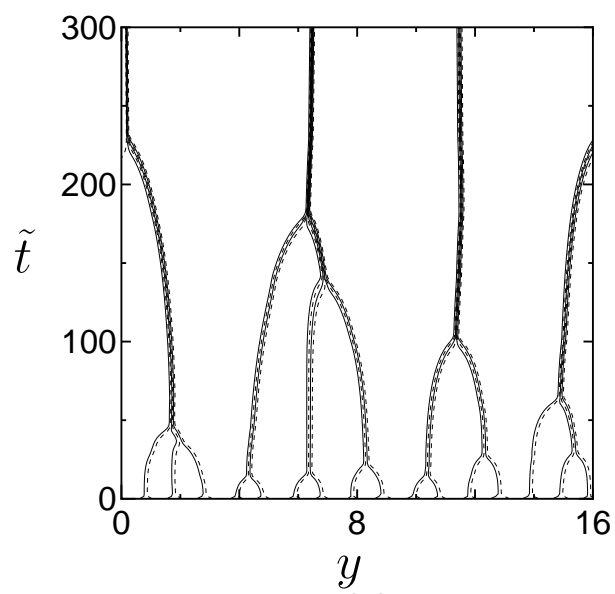

(a)

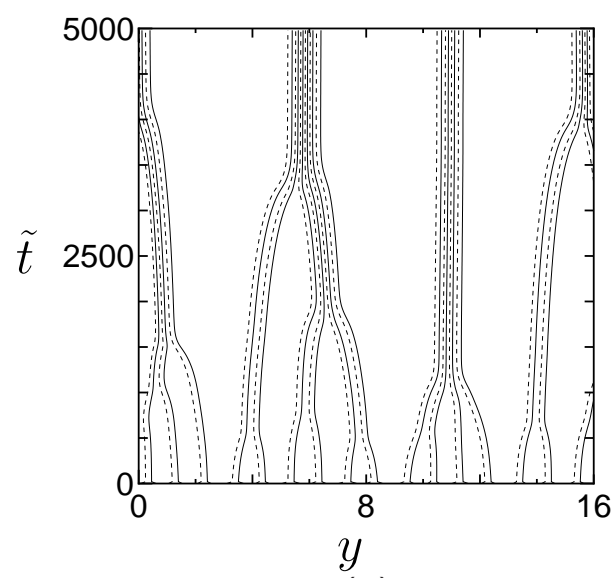

(b)

FIG. 2: Time evolution of step position. The step distance $l$ is larger than $D_{\mathrm{A}} / K$ and $D_{\mathrm{B}} / K$. The drift direction is (a) step-down drift and (b) step-up, and the drift velocities satisfy $|f l|=0.1$. The number of steps is 32 and the system size is 16 with the periodic boundary condition. $\tilde{A}_{\tilde{\nu}}=5 \times 10^{-3}$ with $\tilde{\nu}=1, D_{\mathrm{A}} / K l=0.2, D_{B} / K l=0.02$ and $\tilde{t} \equiv \Omega c_{\mathrm{eq}}^{0} t$.

Figure 2 represents the time evolution of step positions with $D_{\mathrm{A}} / K \ll l \ll f^{-1}$. This is the case in which the diffusion is slow and controls the time evolution. Solid lines represent the evolution of the positions of $S_{B}$ and dotted lines represent $\mathrm{S}_{\mathrm{A}}$. The characteristic length $f^{-1}$ is $f^{-1}=200$ and the scaled time $\tilde{t}$ is given by $\tilde{t} \equiv \Omega c_{\mathrm{eq}}^{0} t$. The initial step distance is about the same, but with a random fluctuation. In the initial stage, the step pairing occurs irrespective of the drift direction. Large terraces are $\mathrm{T}_{\mathrm{A}}$ with step-down drift (Fig. 2(a)) and $\mathrm{T}_{\mathrm{B}}$ with step-up drift (Fig. 2(b)), which agrees with eq. (7) and the previous analysis [4, 5, 6].

In a late stage, large bunches appear irrespective of the drift direction. The type of large terraces is the same as in the initial stage. Free single steps or pairs do not exist on the large terraces and the bunches grow via coalescence of small bunches. Though the formation process of large bunches is similar in both cases, the step density with step-down drift is higher than that with step-up drift, and the growth is much faster. The ratio of the growth rate of step bunches is larger than the ratio of the diffusion coefficients. In the numerical study of Natori et al. [5, 6], the formation of large bunches with step-up drift did not occur in contrast to our simulation. For the very slow growth rate of step bunches, their simulation time might be too short to produce large bunches.

Figure[3represents the step bunching with $l \ll D_{B} / K$, i.e., the step kinetics-contral case. The amplitude of the initial fluctuation is the same as that in Fig. 2 When the drift is in the step-down direction (Fig. 31(a)), the equidistant step train is unstable and the step bunching occurs. The terrace type between step bunches is determined by the initial fluctuation and both types of large terraces coexist. When the drift is in the step-up direction (Fig. 3(b)), the step bunching does not seem to occur. The results are very different from Fig. 2]

To find the reason that the step behavior changes with the kinetic coefficients, we analyse the change of alternating terrace width. We assume the width of $T_{\mathrm{A}}$ is $l_{2 n}=l+\Delta l / 2$ and that of $T_{\mathrm{B}}$ is $l_{2 n-1}=l-\Delta l / 2$. Figure 4 represents the time derivative of $\Delta l$ for large kinetic coefficients. Parameters are the same as in Fig. 2. With step-down drift (Fig. 4 (a)), the initial vicinal face $\Delta l=0$ is unstable and $\Delta l$ increases up to the stable fixed point near $\Delta l=2 l$. The surface consists of large $T_{\mathrm{A}}$ terraces and small $T_{\mathrm{B}}$ terraces, i.e., tight step pairs. With stepup drift (Fig. 4(b)), the initial stage is also unstable and $\Delta l$ decreases to the stable fixed point near $\Delta l=-2 l$. The surface consists of small $T_{\mathrm{A}}$ terraces and large $T_{\mathrm{B}}$ terraces. The results agree with the initial pairing stage of Fig. 2

Figure 5 represents the time derivative of $\Delta l$ for small kinetic coefficients. With step-down drift (Fig. 5]a)), there are three fixed points. The fixed point (open circle) near the center is unstable and two other fixed points (filled circles) are stable. Since the unstable fixed point is very close to $\Delta l=0$, the final stage is not unique if the initial fluctuation is included. In Fig. [3(a), the initial random fluctuation is not negligible and some terraces move to the fixed point with positive $\Delta l$ and two types of terraces coexist.

With step-up drift, there is only one stable fixed point 


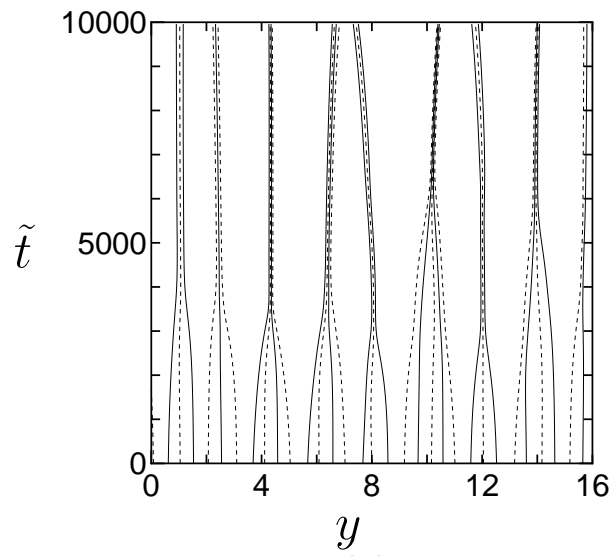

(a)

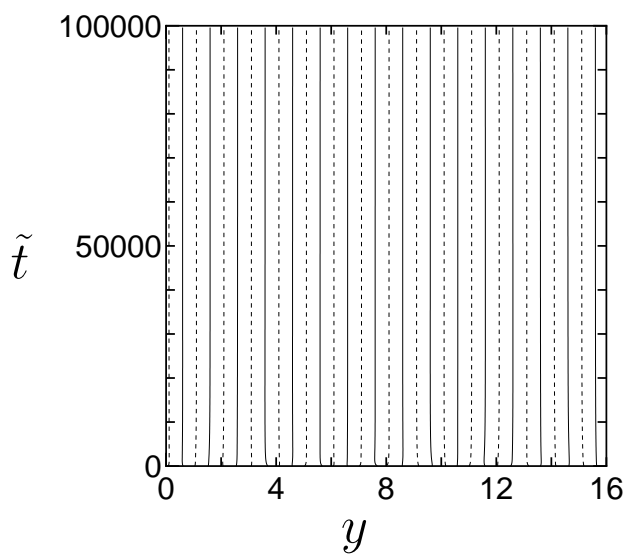

(b)

FIG. 3: Time evolution of step position with step distance $l \ll D_{\mathrm{A}} / K, D_{\mathrm{B}} / K$ with (a) step-down drift and (b) step-up drift. The number of steps is 32 and the system size is 16 with the periodic boundary condition. $\tilde{A}_{\tilde{\nu}}=4 \times 10^{-3}$ with $\tilde{\nu}=1,|f l|=0.1, D_{\mathrm{A}} / K l=200$ and $D_{\mathrm{B}} / K l=20$.

with a small negative $\Delta l$. From the condition $V_{m}$ in eq. (6), the difference of step distance $\Delta l$ in the fixed point near $\Delta l=0$ is given by

$$
\frac{\Delta l}{l}=\frac{K l\left(D_{B}-D_{A}\right)}{2 D_{A} D_{B}},
$$

where we neglected the step-step repulsive interaction. The sign of $\Delta l$ is determined by the diffusion coefficients and independent of the drift direction as seen in Fig. 3. (b).

\section{B. Step bunching induced by the evaporation}

Since the experiments [1, 2] were carried out at high temperatures, the evaporation of adatoms may not be negligible for the step bunching.

We first neglect the drift of adatoms and see if the step bunching occurs with the evaporation. For simplicity, we consider the limit of the fast step kinetics, $K \rightarrow \infty$. If

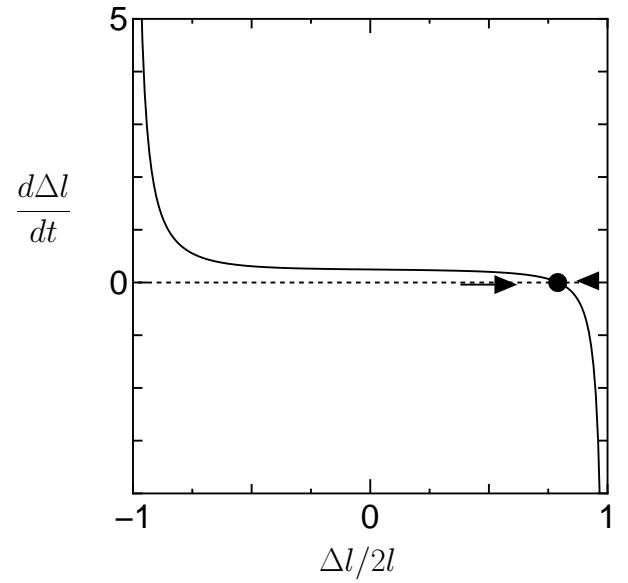

(a)

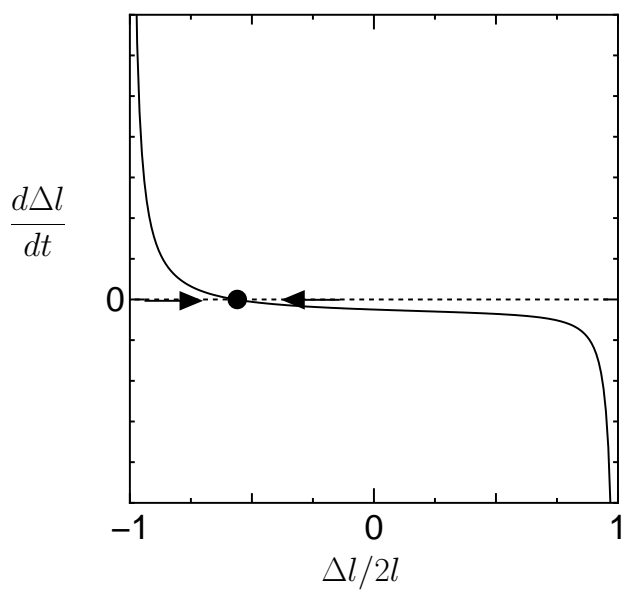

(b)

FIG. 4: Time derivative of the terrace width change $\Delta l$ with (a) step-down drift and (b) step-up drift. Parameters are the same as in Fig. 2

the step repulsion is absent, the step velocity is given by

$$
\frac{V_{m}}{\Omega c_{\mathrm{eq}}^{0}}=-\frac{D_{m}}{x_{m}} \tanh \frac{l_{m}}{2 x_{m}}-\frac{D_{m-1}}{x_{m-1}} \tanh \frac{l_{m-1}}{2 x_{m-1}},
$$

where $x_{m}=\sqrt{D_{m} \tau}$ is the surface diffusion length in the $m$ th terrace. With the same step distance $l$, the step velocity is given by

$$
\begin{aligned}
V_{2 n} & =V_{2 n-1} \\
& =-\Omega c_{\mathrm{eq}}^{0}\left[\frac{D_{\mathrm{A}}}{x_{\mathrm{A}}} \tanh \frac{l}{2 x_{\mathrm{A}}}+\frac{D_{\mathrm{B}}}{x_{\mathrm{B}}} \tanh \frac{l}{2 x_{\mathrm{B}}}\right]
\end{aligned}
$$

where $x_{\mathrm{A}}\left(x_{\mathrm{B}}\right)$ represents the surface diffusion length in $T_{\mathrm{A}}\left(T_{\mathrm{B}}\right)$ and the equidistant train of steps is a steady state. When the terrace widths change alternately and are given by $l_{2 n}=l+\Delta l / 2$ and $l_{2 n-1}=l-\Delta l / 2$, from eq. (10) the time evolution of $\Delta l$ is given by

$$
\frac{1}{2} \frac{d \Delta l}{d t}=V_{2 n}-V_{2 n-1}=0
$$




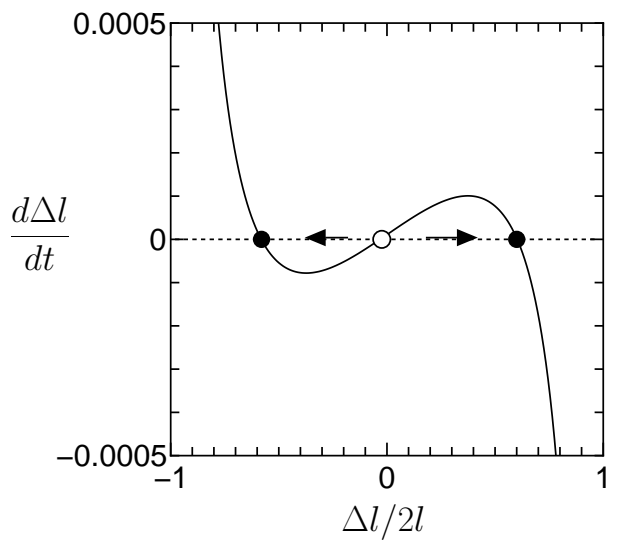

(a)

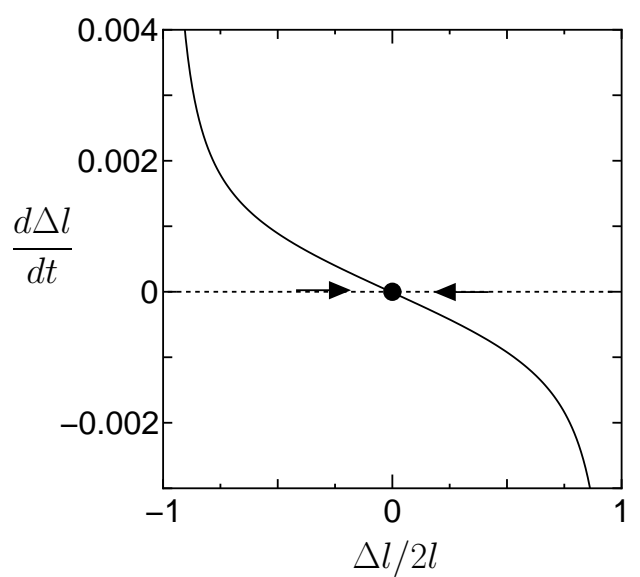

(b)

FIG. 5: Time derivative of the terrace width change $\Delta l$ with (a) step-down drift and (b) step-up drift. Parameters are the same as in Fig. 3

The vicinal face is marginal to the perturbation. If the repulsive interaction is taken into account, the vicinal face is stable and the step pairing does not occur. With the alternation of diffusion coefficient, the evaporation alone does not cause the step bunching.

When both the drift and the evaporation are present, the step velocity is given by

$$
\begin{aligned}
\frac{V_{m}}{\Omega} & =\frac{\left(D_{m-1}-D_{m}\right) f c_{m}}{2} \\
& -\frac{D_{m-1} \alpha_{m-1}\left(c_{m} \cosh \alpha_{m-1} l_{m-1}-e^{-f l_{m-1} / 2} c_{m-1}\right)}{\sinh \alpha_{m-1} l_{m-1}} \\
& -\frac{D_{m} \alpha_{m}\left(c_{m} \cosh \alpha_{m} l_{m}-e^{-f l_{m} / 2} c_{m+1}\right),}{\sinh \alpha_{m} l_{m}}
\end{aligned}
$$

The parameter $\alpha_{m}$ is defined by

$$
\alpha_{m}=\frac{1}{2} \sqrt{f^{2}+\frac{4}{x_{m}^{2}}} .
$$

$\alpha_{m}^{-1}$ is the characteristic length of the diffusion field in the $m$ th terrace.

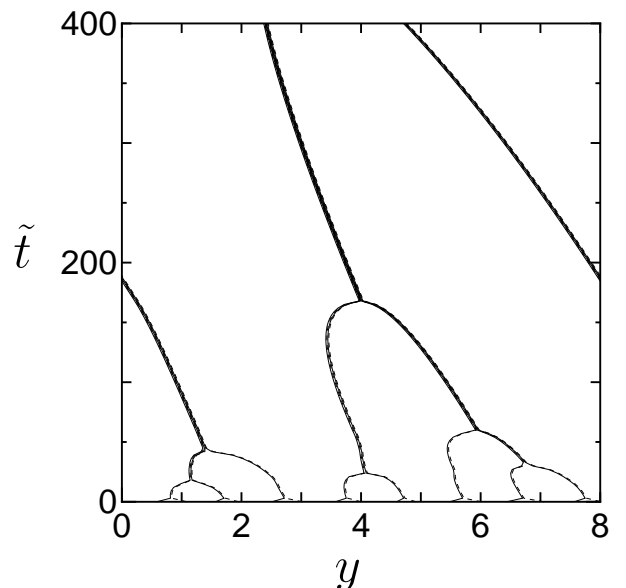

(a)

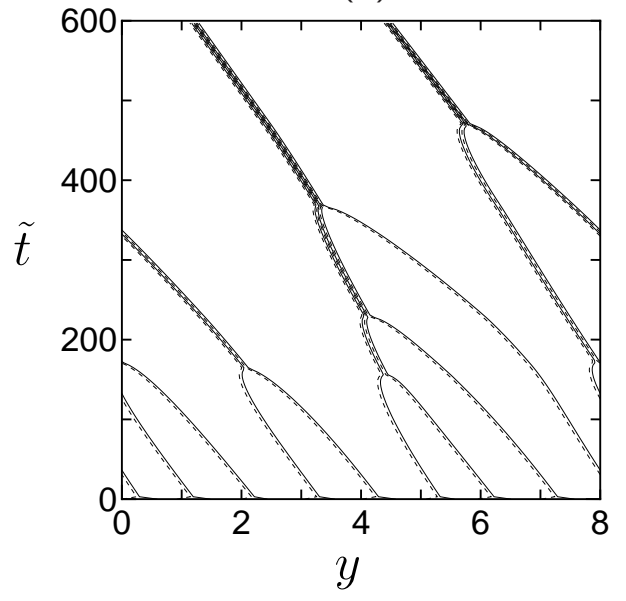

(b)

FIG. 6: Time evolution of step position with evaporation with (a) step-down drift and (b) step-up drift. The number of steps is 16 and system size is 8 . The diffusion coefficients are $D_{B}=2$ and $D_{A}=10$. Other parameters are $f=0.1$ $\nu \Omega A / k_{\mathrm{B}} T=10^{-4}$ with $\nu=1$, and $\tau=50$.

We carry out numerical integration of eq. (13). The time evolution of step positions (Figure 6] shows the drift-induced step bunching with the evaporation, where the characteristic length scale in $\mathrm{T}_{\mathrm{A}}$ is $\alpha_{2 n}^{-1}=14.9$ and that in $\mathrm{T}_{\mathrm{B}}$ is $\alpha_{2 n-1}^{-1}=8.9$. Irrespective of the drift direction, very fast pairing of receding steps occurs at the initial stage. The type of large terraces is the same as in Fig 2 and not changed by the evaporation.

Large bunches appear by coalescence of small bunches. With step-down drift, coalescence of step bunches occurs successively, but the time interval of the coalescence increases with increasing the terrace width between bunches (Fig. 6(a)). With step-up drift, coalescence of step pairs does not occur until $\tilde{t} \approx 150$. However, once the coalescence of step pairs starts, the interval does not seem to increase much (Fig. [6 (b)). The difference of the growth rate of bunches is much smaller than that without the evaporation. 


\section{STEP BUNCHING WITH ALTERNATION OF KINETIC COEFFICIENT}

In a $\mathrm{Si}(001)$ vicinal face, not only diffusion coefficients but also the type of steps changes alternately. In this section, we study the step bunching due to the alternation of kinetic coefficient and compare the result with Sec. III

\section{A. Step bunching induced by the drift}

To focus on the alternation of step kinetics, we neglect the alternation of diffusion coefficients. First, we neglect the evaporation. Without the evaporation, the step velocity is given by

$$
\begin{aligned}
V_{m} & =\frac{\Omega D_{\mathrm{s}} f K_{m} K_{m-1}\left(e^{f l_{m-1}} c_{m-1}-c_{m}\right)}{K_{m-1}\left(D_{\mathrm{s}} f-K_{m}\right)+K_{m}\left(D_{\mathrm{s}} f+K_{m-1}\right) e^{f l_{m-1}}} \\
& -\frac{\Omega D_{\mathrm{s}} f K_{m} K_{m+1}\left(e^{f l_{m}} c_{m}-c_{m+1}\right)}{K_{m}\left(D_{\mathrm{s}} f-K_{m+1}\right)+K_{m+1}\left(D_{\mathrm{s}} f+K_{m}\right) e^{f l_{m}}}(15)
\end{aligned}
$$

where $D_{\mathrm{s}}$ is the diffusion coefficient.

In a vicinal face with equidistant steps, the step velocities are given by

$$
\begin{aligned}
V_{2 n} & =-V_{2 n-1} \\
& =\frac{\Omega D_{s} K_{A} K_{B} c_{\mathrm{eq}}^{0} f^{2}\left(e^{f l}-1\right)^{2}\left(K_{B}-K_{A}\right)}{g_{\mathrm{A}} g_{\mathrm{B}}},
\end{aligned}
$$

where $g_{\mathrm{A}}$ and $g_{\mathrm{B}}$ are

$$
\begin{aligned}
& g_{\mathrm{A}}=\left[K_{A}\left(D_{\mathrm{s}} f-K_{B}\right)+K_{B}\left(D_{\mathrm{s}} f+K_{A}\right) e^{f l}\right], \\
& g_{\mathrm{B}}=\left[K_{B}\left(D_{\mathrm{s}} f-K_{A}\right)+K_{A}\left(D_{\mathrm{s}} f+K_{B}\right) e^{f l}\right] .
\end{aligned}
$$

Since the kinetic coefficient $K_{B}$ is larger than $K_{A}, S_{\mathrm{A}}$ steps advance and $S_{\mathrm{B}}$ steps recede. Alternation of large $\mathrm{T}_{\mathrm{A}}$ terraces and small $\mathrm{T}_{\mathrm{B}}$ terraces is expected by formation of step pairs.

When the kinetic coefficient $K_{B}$ is large, $D_{\mathrm{S}} / K_{\mathrm{B}} \ll l$, time evolution of step positions is shown in Fig. [7 With step-down drift (Fig. 7(a)), most steps form pairs and large $T_{\mathrm{A}}$ terraces appear in the initial stage, but formation of triplets also occurs and a large $T_{\mathrm{B}}$ is produced. In a late stage, all large terraces become $T_{\mathrm{A}}$ after coalescence of small bunches. With step-up drift (Fig. Z(b)), the pairing does not seem to occur.

Figure 8 represents the growth rate of $\Delta l$, which is defined in the same way as in Sec. IIIA With step-down drift, an unstable fixed point with a small positive $\Delta l$ and two stable fixed points with a large amplitude of $|\Delta l|$ are present. From the condition $V_{m}=0$ in eq. (15), the difference of terrace width at the fixed point near $\Delta l=0$ is given by

$$
\frac{\Delta l}{l}=\frac{K_{A}-K_{B}}{K_{A}+K_{B}} f l,
$$

where we have used $f l \ll 1$ and neglected the step-step repulsion. In eq. (9) $\Delta l$ is determined only by structural

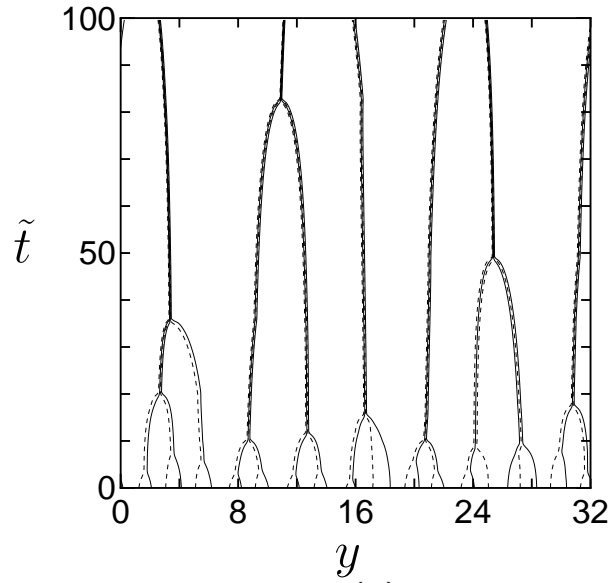

(a)

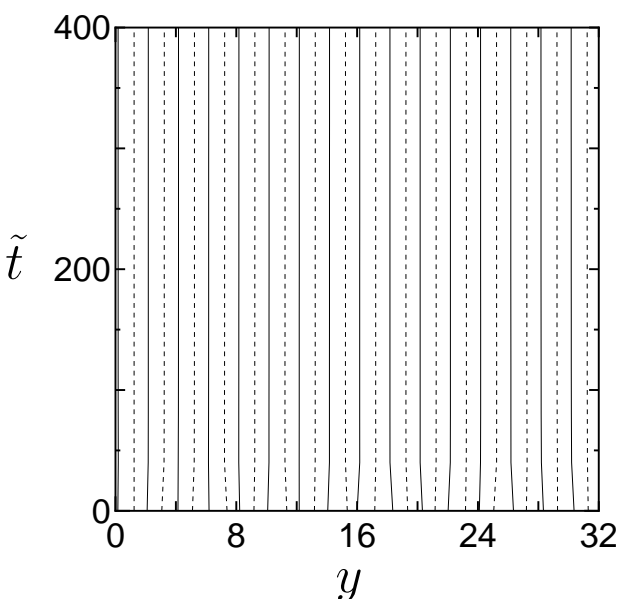

(b)

FIG. 7: Time evolution of step position with alternating change of kinetic coefficient with step distance $D_{\mathrm{s}} / K_{\mathrm{A}} \ll 1$. Drift is in (a) step-down direction and (b) the step-up direction. The number of steps is 32 and the system size is 32 with the periodic boundary condition. Parameters are $\tilde{A}_{\tilde{\nu}}=2 \times 10^{-2}$ with $\tilde{\nu}=1,|f l|=0.2, D_{s} / K_{\mathrm{A}} l=1.0 \times 10^{-2}$ and $D_{s} / K_{\mathrm{B}} l=1.0 \times 10^{-3}$.

parameters and independent of the drift, but in eq. (18) $\Delta l$ depends on the drift and changes the sign with the drift direction. Expect for the sign of $\Delta l$ in the unstable fixed points, the form of $d \Delta l / d t$ is the same as in Fig. [5] The initial vicinal face moves to the steady state with the large negative $\Delta l$ if the initial fluctuation is small, but it can move to the other steady state with large $\Delta l$ if the initial fluctuation is large. In Fig. Z(a), formation of a large $T_{\mathrm{B}}$ in the initial stage is due to a large fluctuation of terrace width.

With step-up drift, there is only one fixed point with a negative $\Delta l$. The steady state is stable. Since the difference of terrace width $\Delta l$ is small, the surface looks like the original vicinal face in Fig. Z7(b).

With the alternation of diffusion coefficient, the form of $d \Delta l / d t$ changes with decreasing the kinetic coefficients 


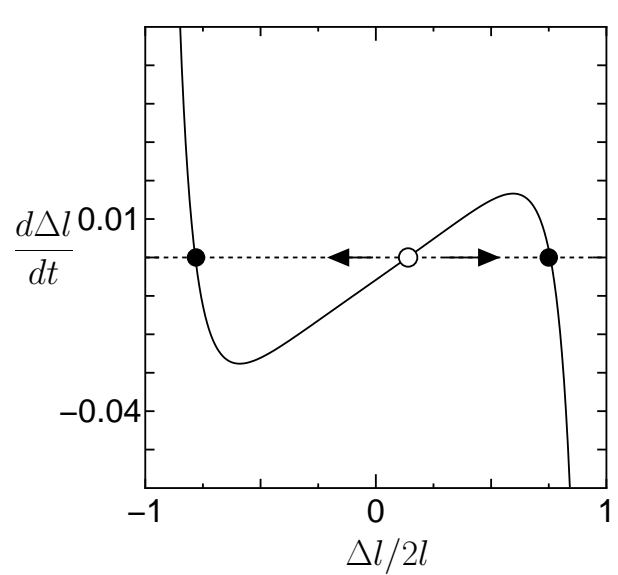

(a)

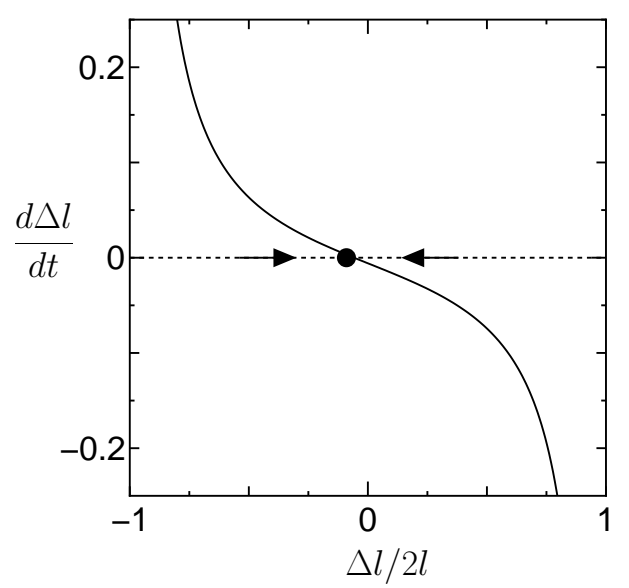

(b)

FIG. 8: Time derivative of the terrace width change with (a) step-down drift and (b) step-up drift. Parameters are the same as in Fig. 7

(Figs. 4 and 5). On the other hand, with the alternation of kinetic coefficient, the form of $d \Delta l / d t$ does not change even if $D_{s} / K$ changes. Thus the bunching behavior is insensitive to the ratio $D_{s} / K l$.

\section{B. Step bunching induced by the evaporation}

If the evaporation is present and the drift is absent, the step velocity is given by

$$
\begin{aligned}
& \frac{x_{\mathrm{s}} V_{m}}{\Omega D_{\mathrm{s}}}= \\
& -\frac{\left(\lambda_{m+1} \sinh l_{m} / x_{\mathrm{s}}+\cosh l_{m} / x_{\mathrm{s}}\right) c_{n}-c_{n+1}}{h_{m+1}\left(l_{m}\right)} \\
& -\frac{\left(\lambda_{m-1} \sinh l_{m-1} / x_{\mathrm{s}}+\cosh l_{m-1} / x_{\mathrm{s}}\right) c_{n}-c_{n-1}}{h_{m}\left(l_{m-1}\right)}(19)
\end{aligned}
$$

where $\lambda_{m}=D_{\mathrm{s}} / K_{m} x_{\mathrm{s}}$, and $h_{m}(l)$ is

$$
\begin{aligned}
h_{m}(l)= & \left(\lambda_{m-1} \lambda_{m}+1\right) \sinh l / x_{\mathrm{s}} \\
& +\left(\lambda_{m}+\lambda_{m-1}\right) \cosh l / x_{\mathrm{s}} .
\end{aligned}
$$

Due to the evaporation, both $S_{\mathrm{A}}$ steps and $S_{\mathrm{B}}$ steps recede. When the steps are equidistant, the difference of step velocities is given by

$$
V_{2 n}-V_{2 n-1}=\frac{\left(\lambda_{\mathrm{A}}-\lambda_{\mathrm{B}}\right) \tanh l / x_{\mathrm{s}}}{\left(1+\lambda_{\mathrm{A}} \lambda_{\mathrm{B}}\right) \tanh l / x_{\mathrm{s}}+\left(\lambda_{\mathrm{A}}+\lambda_{\mathrm{B}}\right)} .
$$

Since $\lambda_{\mathrm{A}}$ is smaller than $\lambda_{\mathrm{B}}, S_{\mathrm{B}}$ recedes faster than $\mathrm{S}_{\mathrm{A}}$. The vicinal face is unstable, and large $\mathrm{T}_{\mathrm{B}}$ terraces and small $\mathrm{T}_{\mathrm{A}}$ terraces appear by step pairing.

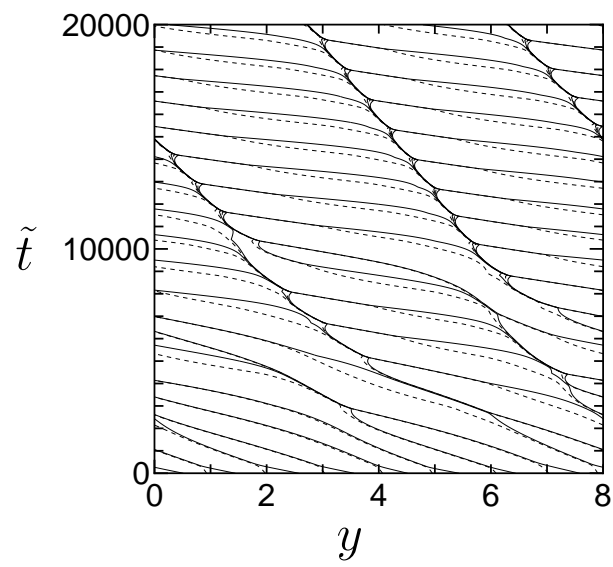

FIG. 9: Time evolution of step position. $x_{\mathrm{s}}=15, K_{\mathrm{A}}=$ $0.125, K_{\mathrm{B}}=1.0, D_{\mathrm{s}}=1.0$. The number of step is 16 and the system size is 8 with the periodic boundary condition. $\tilde{A}_{\tilde{\nu}}=\times 10^{-4}$ with $\tilde{\nu}=1$.

Figure 9 represents the time evolution of step positions, which is obtained by numerical integration of eq. (19). In the simulation, the difference of kinetic coefficients is very large. In very early stage, pairing of steps occurs and large $T_{\mathrm{B}}$ terraces appear. Via coalescence of step pairs, step bunches appear. The type of large terrace is the same as that in the step bunching induced by the drift. In large terraces, isolated step pairs are present. When a collision between a step bunch and a step pair occurs, another step pair leaves from the upper side of the step bunch. A step pair breaks into single steps, but only temporarily. Repeating of such collisions is seen in other bucnhing systems 14, 15]. Here a step pair is the fundamental unit.

\section{SUMMARY AND DISCUSSION}

In this paper, we studied the drift-induced step bunching with the alternation of structural parameters: the diffusion coefficient and the kinetic coefficient.

With the alternation of diffusion coefficients on consecutive terraces, the step bunching occurs irrespective of the drift direction if the kinetic coefficients are large. The type of large terraces is determined by the drift direction. When the kinetic coefficients are small, the step bunching occurs with step-down drift and the initial fluctuation of step distance influences the type of step pairs. 
The growth rate of bunches is much faster with the stepdown drift, but the difference of growth rates decreases with the evaporation. Without drift of adatoms, the evaporation does not induce bunching.

With the alternation of kinetic coefficient at consecutive steps, the step bunching occurs with the step-down drift and does not occur with the step-up drift. The type of large terraces is influenced by the initial fluctuation of step distance. The evaporation induces step bunching even if the drift is absent, in contrast to the diffusion coefficient case.

In the experiments 1, 2], the type of terraces between bunches changes with the drift direction when the initial step distance is large. From our result we may conclude that the alternation of diffusion coefficient is essential to the bunching and that of kinetic coefficient is not so important. Also the kinetic coefficients are large if the steps are impermeable. Impermeable steps with large kinetic coefficients are effectively equivalent to the permeable steps [7, 17]. However, when the step distance is smaller than the critical value, the step bunching occurs only with the step-up current in the experiment [1], which is not explained by our model. In the previous studies [17, 18], the dift direction to cause the step bunching on a $\mathrm{Si}(111)$ vicinal face is affected by the step permeability. In a similar way, the disagreement in a $\mathrm{Si}(001)$ vicinal face may be explained by the step permeability, which remains to be explored.

The difference of growth rate of step bunches as the change of current direction is very small in the experiment [2]. The evaporation may play an important role, but the difference does not vanish only with the evaporation. In the Monte Carlo simulation [7], in which steps are permeable, a similar behavior has been observed. We still do not undestand the origin of such a large difference.

\section{Acknowledgments}

M.S. thanks to N. Miura for helping simulations. This work was supported by a Grant-in-Aid from the Japanese Society for the Promotion of Science.
[1] L. V. Litvin, A. B. Krasilnikov and A. V. Latyshev, Surf. Sci. Lett. 244 (1991) L121.

[2] A. V. Latyshev, L. V. Litvin, A. L. Aseev, Appl. Surf. Sci. 130 (1998) 139.

[3] M. Ichikawa and T. Doi, Appl. Phys. Lett. 60, 1082 (1992).

[4] S. Stoyanov, Jpn. J. Appl. Phys. 29 (1990) L659.

[5] A. Natori, H. Fujimura and H. Yasunaga, Jpn. J. Appl. Phys. 31 (1992) 1164.

[6] A. Natori, H. Fujimura and M. Fukuda, Appl. Surf. Sci. 60/61 (1992) 85.

[7] M. Sato, M. Uwaha and Y. Saito, J. Cryst. Growth 237239, 43 (2002).

[8] M. Ozdemir, A. Zangwill, Phys. Rev. B 45 (1992) 3718.

[9] W. W. Mullins, in: W. D. Robertson, N. A. Gjostein Eds., Metal Surfaces, Metall. Soc. AIME, Metals Park
(1963).

[10] M. Uwaha, Phys. Rev. B 46 (1992) 4364.

[11] O. L. Alerhand, D. Vanderbilt, R. D. Meade, and J. D. Joannopoulos, Phys. Rev. Lett. 61 (1988) 1973.

[12] H. Kahara and K. Yagi, Jpn. J. Appl. Phys. 28 (1989) 1042.

[13] M. Sato and M. Uwaha, J. Phys. Soc. Jpn. 67 (1998) 3675 .

[14] M. Sato and M. Uwaha, Surf. Sci. 442 (1999) 318.

[15] M. Sato and M. Uwaha, Phys. Rev. B 51 (1995) 11172.

[16] S. Tanaka, N. C. Bartelt, C. C. Umbach, R. M. Tromp, and J. M. Blackley, Phys. Rev. Lett. 78 (1997) 3342.

[17] M. Sato, M. Uwaha, and Y. Saito, Phys. Rev. B 62 (2000) 8452 .

[18] S. Stoyanov, Surf. Sci. 416 (1998) 200. 